\title{
EL ASENTAMIENTO DE POBLACIÓN EN LAS CAÑADAS DEL TEIDE. ESTUDIO HISTÓRICO DE SU OCUPACIÓN (1910-2019)
}

\author{
Fernando de Ory Ajamil* \\ Universidad de La Laguna
}

\section{Resumen}

Este trabajo pretende analizar las causas y consecuencias de los asentamientos periódicos y estables de población que se produjeron en Las Cañadas del Teide, desde la primera década del siglo xx hasta la actualidad. Se trata de un estudio histórico sobre un territorio que pasó de ser ignorado a codiciado por diferentes motivos e intereses: terapéuticos, turísticos, económicos y municipales. Se describirá el modus vivendi de los enfermos que acudieron a Las Cañadas del Teide para la curación de diversas enfermedades. Se expondrá el reiterado y público debate en relación con la idoneidad del clima y el emplazamiento para tales fines, y la posición de las autoridades públicas al respecto. Mientras las iniciativas oficiales por parte de las autoridades isleñas se frustraron hasta la creación del Sanatorio Antituberculoso de Santa Cruz, en el año 1943, la población afectada hizo un uso continuo de aquel territorio de modo espontáneo, clandestino y hasta furtivo. La creación del Parque Nacional del Teide, en el año 1954, decretó la prohibición de tales usos pero no acabó con la aparición de otras tantas construcciones, estas sí estables y permanentes, que aún hoy están presentes.

Palabras clave: Las Cañadas del Teide, La Orotava, Vilaflor, Alemania, tuberculosis, clima, sanatorio, funicular, chozas.

\section{POPULATION SETTLEMENTS IN LAS CAÑADAS DEL TEIDE. HISTORICAL STUDY OF ITS OCCUPATION}

\section{Abstract}

This paper aims to analyze the causes and consequences of the periodic and stable population settlements that took place in Las Cañadas del Teide, from the first decade of the twentieth century to the present. This is a historical study on a territory that went from being ignored to being coveted for different reasons and interests: therapeutic, tourist, economic and municipal. The modus vivendi of the patients who came to Las Cañadas del Teide for the cure of various diseases will be described. The repeated and public debate on the suitability of the climate and location for these purposes, and the position of public authorities thereon, will be presented. While the official initiatives of the island authorities were thwarted until the establishment of the Sanatorio Antituberculoso de Santa Cruz, in 1943, the affected population made a continuous use of that territory, spontaneous, clandestine and even furtive. The creation of the Parque Nacional del Teide, in the year 1954, decreed the prohibition of such uses but did not end with the appearance of many other constructions, these stable and permanent, that are still present today.

Keywords: Las Cañadas del Teide, La Orotava, Vilaflor, Germany, tuberculosis, climate, sanatorium, funicular, huts. 


\section{INTRODUCCIÓN}

La tuberculosis - consunción, tisis, mal del rey, peste blanca o plaga blancaes considerada una de las enfermedades humanas más antiguas. Se trata de una enfermedad infecciosa causada por microbacterias, con gran variedad de cuadros clínicos dependiendo del órgano al que afecte. Se contagia por vía aérea, cuando las personas infectadas tosen, estornudan o escupen. Se contrae, además, debido al debilitamiento del sistema inmunitario por malas condiciones de higiene, alimentación, hábitos de vida y carencia de salud en general. El tratamiento de la tuberculosis es complicado y requiere largos periodos de reposo, dieta y administración de diversos antibióticos.

Actualmente es posiblemente la enfermedad infecciosa más prevalente del mundo. Considerando su forma latente, en la cual no presenta síntomas, se estima que afecta al 33\% de la población mundial. Asimismo, es la segunda causa de muerte a nivel global, y la primera entre las enfermedades infecciosas.

Pues bien, si en el año 1866 Jean Antoine Villemin demostraba que podía contagiarse la enfermedad, en 1882 Robert Koch descubrió el bacilo infeccioso que la provocaba. Fue entonces cuando comenzaron a proliferar los sanatorios para tuberculosos, mejorando su pronóstico y comenzando a cortar la cadena de transmisión. Hermann Brehmer, médico de ideas liberales nacido en Kurtsch (Silesia) en 1826, estaba convencido de que el origen patogénico de la tuberculosis se encontraba en la dificultad del corazón para irrigar correctamente a los pulmones. Con la ayuda de Alexander von Humboldt y a pesar de la oposición inicial del Gobierno prusiano, construye en 1854 el que es considerado el primer sanatorio antituberculoso, en Görbersdorf, Silesia, a 650 metros sobre el nivel del mar. De tal modo comenzaron a construirse los sanatorios a gran altura, basándose en la teoría fisiológica de aumentar el flujo sanguíneo pulmonar, por la taquicardia inducida por la altura. Sin embargo, la evidencia de su eficacia resultó dudosa ${ }^{1}$.

El propio Hermann Brehmer y un paciente suyo, Peter Dettweilwer, fueron los promotores, en el año 1877, de la proliferación de este tipo de asilos para tísicos, inicialmente en Alemania, pero pronto extendidos a toda Europa. Peter Dettweilwer funda en 1876 otro sanatorio en Falkenstein y diez años después publica los resultados de su terapia, en los que confirma la curación completa de 132 pacientes de los 1022 que habían ingresado y permanecido más de un mes en su institución. Edward L. Trudeau fundaría en 1884 el sanatorio Saranac Lake, el primero en Estados Unidos. Su máxima propagación tendría lugar en la segunda mitad del siglo XIX $\mathrm{y}$ en los inicios del siglo $\mathrm{xx}$, dando nombre a una etapa de la medicina moderna: la

* Doctor en Historia Contemporánea por la Universidad de La Laguna. Profesor de Historia de la Escuela de Adultos y Mayores de la Universidad de La Laguna (ULL). Correo electrónico: fernandodeory@gmail.com.

${ }^{1}$ Báguena Cervellera, María José (1992): La Tuberculosis y su historia. Fundación Uriach. 
era del movimiento sanatorial. Sir Robert Phillip crea en 1887 el primer dispensario antituberculoso del Reino Unido, en Londres, y otro en Edimburgo en $1889^{2}$.

En 1895 Wilhelm Röntgen descubre los rayos X, lo que permitió diagnosticar y seguir la evolución de la enfermedad, y aunque faltaran casi cincuenta ańos para el hallazgo de un tratamiento farmacológico eficaz, la incidencia y la mortalidad comienzan a caer. En 1921 fue descubierta la vacuna BCG por Albert Calmette y Camile Guérin, de cuyas iniciales toma el nombre (BCG = bacilo de CalmetteGuérin). En 1944, en plena guerra mundial, Albert Schatz y Selman Waksman descubren la estreptomicina, con una eficacia limitada pero superior a los tratamientos dietéticos y «balneoterápicos» empleados hasta ese momento. Este hito es considerado como el comienzo de la era moderna de la tuberculosis, aunque la verdadera revolución se produce pocos años después, en 1952, con el desarrollo de la isoniacida, el primero de los antibióticos específicos que convertirán a la tuberculosis en una enfermedad curable en la mayoría de los casos. La aparición de la rifampicina en la década de los setenta acortó notablemente los tiempos de curación, lo que hizo disminuir el número de casos nuevos de manera importante hasta la década de los ochenta.

\section{PRIMEROS SANATORIOS O «HEALTH RESORTS» EN CANARIAS}

Una vez que el médico y naturalista William Anderson, tripulante en el tercer viaje de James Cook por los mares australes (1776-1779), escribiera que «el aire y el clima de Tenerife son notablemente sanos y particularmente apropiados para prestar alivio a enfermedades tales como la tuberculosis», ya no cesó la búsqueda de emplazamientos en las islas atlánticas - primero en las portuguesas de Azores y Madeira, y luego entre Gran Canaria y Tenerife-. En su libro The sanative influence of climate, el también británico James Clark, tras visitar Tenerife en la década de 1820, recomendaba particularmente La Orotava y Vilaflor como emplazamientos adecuados para la curación de la tuberculosis.

Sin embargo, el renombrado médico tinerfeño Víctor Pérez Ventoso describía de manera tan pesimista como detallada la situación al comenzar la década de 1880, y así,

de algunos ańos á esta parte se preconiza altamente, sobre todo en Inglaterra, la isla de Tenerife, del grupo de las Canarias, como residencia propia para los tísicos... no hay en toda la Isla, ni en La Orotava, ni en otro punto, una sola instalación conveniente para los enfermos; los recursos alimenticios son completamente defectuosos bajo el punto de vista de su calidad; y no hay razón alguna para esperar que esto cambie; porque los indígenas rechazan con una hostilidad cercana á la indignación la idea de adaptar su país para residencia de los tísicos. No quieren por ningún precio oír hablar de semejantes

2 Oriol Anguera, J. y A. (1944): Historia de la tuberculosis. (Ensayos de fisiología colectiva). Salvat Editores, Barcelona. 
transformaciones... La cuestión está, pues, juzgada y la isla de Tenerife tiene que ser borrada de la lista de estaciones médicas, á pesar de las ventajas excepcionales de su clima, polvo y mosquitos aparte ${ }^{3}$.

Desde el 20 de septiembre de 1887, el Diario de Tenerife publicó por capítulos la traducción del trabajo de Ernest Hart, bajo el título «Una excursión en invierno a las Islas Afortunadas», donde el médico británico exponía este y otros criterios, así como las ventajas del clima del archipiélago canario sobre el de Madeira y otros lugares hasta entonces frecuentados por los enfermos de pecho de las islas Británicas.

A partir de la creación de la «Compañía de Hoteles y Sanatorium del valle de la Orotava», en el Puerto de la Cruz en 1886, Canarias presenciará una irrupción hotelera no conocida hasta entonces: La Orotava, Vilaflor, Icod, La Laguna, Güímar, Santa Cruz de Tenerife, así como diversos lugares de Gran Canaria, iban a ser testigos del nacimiento de un buen número de establecimientos hoteleros.

Las investigaciones sobre la tisis discurrieron según dos corrientes tan bien definidas como contrapuestas: los británicos postulaban las propiedades terapéuticas y curativas de la hidroterapia marina, mientras los germanos confiaban en el clima de alta montańa para el tratamiento de la tuberculosis, tras constatar la ausencia de tal enfermedad entre los habitantes de esos territorios.

En cualquier caso, el origen del turismo en Canarias formó un binomio con su clima y su desarrollo inicial lo fue bajo el apelativo de "turismo terapéutico». Este se organizó para que las clases medias británicas escaparan de las condiciones de vida derivadas de la Revolución Industrial en las metrópolis británicas. Siendo este un tema suficientemente estudiado en la historiografía canaria, nos remitimos a la abundante bibliografía existente sobre el mismo.

\section{LA TUBERCULOSIS EN CANARIAS}

Por lo que se refiere al impacto de la tuberculosis en el archipiélago canario, esta fue una cuestión que interesó y preocupó enormemente a los médicos y autoridades tanto nacionales como locales, en línea con lo expresado por sus colegas europeos. De tal modo, el médico Agustín Bassols y Prim escribía en el año 1888 el más completo trabajo español sobre la cuestión, titulado Climatoterapia española en la tisis pulmonar. Estudio de los diversos puntos que en España, Islas y Baleares y Canarias pueden utilizarse para sanatorios por los tísicos. Pocos años antes, en 1884, el médico lanzaroteño Tomás Zerolo Herrera publicaba en la imprenta de Vicente Bonnet de Santa Cruz de Tenerife su trabajo Orotava-Vilaflor. Estaciones Sanitarias de Tenerife, y cinco años después su Climatoterapia de la tuberculosis pulmonar. En el mismo año el médico grancanario Gregorio Chil y Naranjo sacaba a la luz sus Estudios históricos, climatológicos y patológicos de las Islas Canarias. Y aún es posible

3 PÉrez, V. (1881): «Tenerife como estación médica». Revista de Canarias t. III, pp. 115-116. 
considerar algún estudio más avanzado en el tiempo como fue el del médico francés Gabriel Belcastel, quien en 1861 publicaba en París su estudio titulado Las Islas Canarias y el Valle de La Orotava, desde el punto de vista médico e higiénico.

En línea con estos trabajos teóricos, prácticamente la totalidad de la prensa isleńa registraba a finales del siglo XIX un alarmante incremento del número de afectados por tuberculosis entre la población canaria. El diario palmero El Pancista citaba ya en 1895 un "aumento de muertes por tuberculosis»" ${ }^{4}$. En 1902 la revista Las Canarias insertaba un artículo de la revista $L a$ Orotava, donde se afirmaba que «las escuelas públicas de niñas están desiertas. Nuestros pueblos y campos están plagados por la tuberculosis, esa terrible enfermedad casi desconocida entre nosotros hasta hace poco, y que hoy en día toma un creciente desarrollo... Y se comprende: el trabajo excesivo y mal reglamentado; largas jornadas de faena, malas noches; y luego el comer aprisa y el levantarse aún de noche para continuar la labor comenzada»s. Canarias fue una de las regiones más cruelmente castigadas por la tuberculosis, aseguraba el diario El Tiempo ${ }^{6}$ en el año 1906.

En 1905-1906, se afirmaba en la revista Las Canarias que «el 16.03\% de nuestros tuberculosos en España, lo eran en Santa Cruz de Tenerife»?. Sirva igualmente como referencia una relación pormenorizada publicada por la dirección general de Sanidad, la cual señalaba que entre 1908 y 1923, «el número de muertos en Espańa por causa de la tuberculosis no bajó de 30000 ninguno de esos años, llegándose a los 40000 en 1918 y años siguientes al de la gripe española, registrándose entonces en Canarias más de 2000 muertos por esta epidemia» ${ }^{8}$.

\section{LAS CAÑADAS DEL TEIDE, OBJETIVO CIENTÍFICO Y GEOESTRATÉGICO DE ALEMANIA}

En marzo de 1909 sucede un acontecimiento de capital importancia en el hasta entonces inhabitado y desconocido paisaje de Las Cañadas del Teide: la instalación de un observatorio meteorológico alemán en La Cañada de La Grieta. El establecimiento de dos construcciones portátiles fue posible por el apoyo de la casa imperial alemana y la decidida participación del káiser Guillermo II, ocasionando por tal razón un conflicto diplomático con el gobierno español, no resuelto hasta varios años después?.

\footnotetext{
El Aire. Ed. Idea.

9 Para cualquier ampliación o aclaración remito a mi trabajo Ciencia y diplomacia hispanoalemana en Canarias: el origen del observatorio meteorológico de Izaña (1907-1916). Ed. Edirca, 1995.
}

${ }^{4}$ El Pancista, 04.08.1895.

Las Canarias, 15.02.1902.

${ }^{6}$ El Tiempo, 25.04.1906.

Las Canarias, 05.11.1911.

${ }^{8}$ De Ory Ajamil, Fernando (2004): Ciencia y Presencia Extranjera en Canarias, volumen 

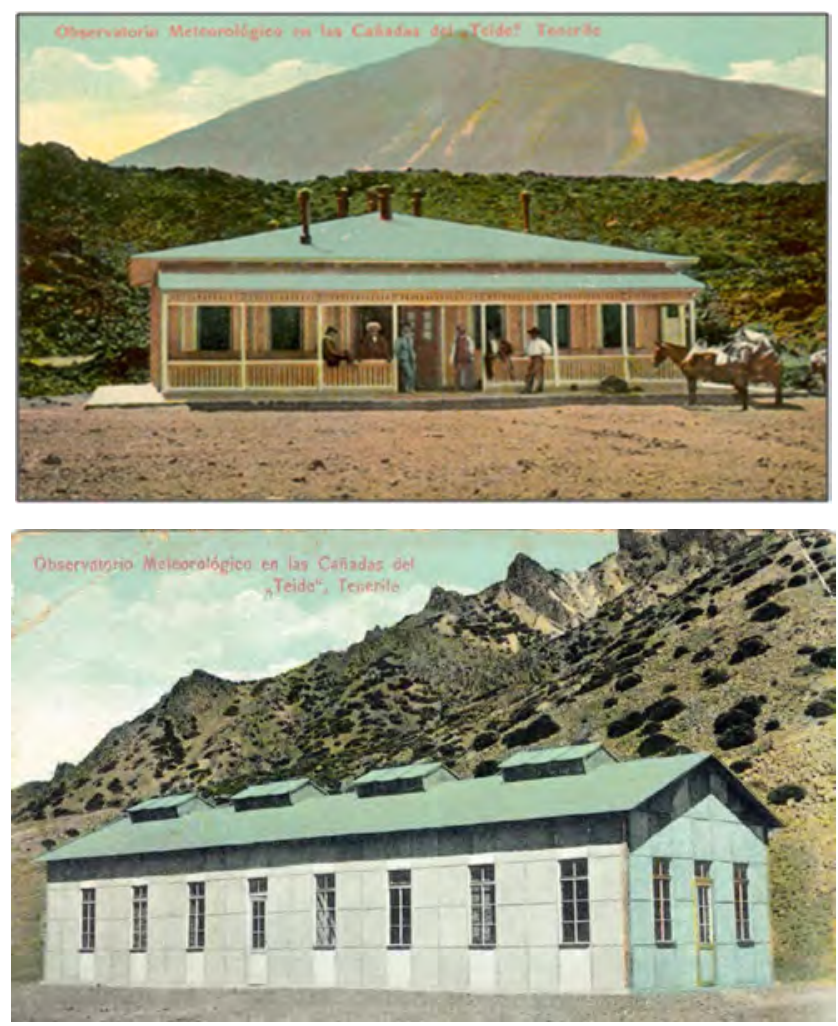

Figuras 1 y 2. El chalet del Káiser Guillermo II de Alemania y el hangar, probable propiedad del conde Ferdinand von Zeppelin. Circa 1910.

La instalación del citado observatorio fue llevada a cabo por el meteorólogo y catedrático de la Universidad de Berlín Hugo Hergesell, consejero privado del káiser y asesor científico del conde Fernando de Zeppelin, y por Gothald Pannwitz, médico militar y reconocido especialista internacional en tuberculosis, fundador de la Unión Internacional contra la Tuberculosis. El interés de la expedición alemana por Las Cañadas del Teide rebasaba el marco estrictamente meteorológico, pues, entre otros recursos, pretendía la explotación del emplazamiento para la construcción de un sanatorio antituberculoso. No en vano, el propio Pannwitz había adquirido en 1907 el Hotel Taoro de La Orotava, que pasó a llamarse Humbodlt Kurhaus.

Del mismo modo que Piazzi Smyth se estableciera durante dos meses de verano en las cumbres de Tenerife en 1856, para comprobar las palabras de Isaac Newton en relación con la idoneidad de los lugares elevados para la instalación de los telescopios, el interés de Pannwitz era llevar a cabo diferentes experiencias para el estudio de la tuberculosis y la influencia del clima de alta montaña en el organismo humano. De tal modo, tras la IX Conferencia Internacional contra la Tuber- 
culosis, celebrada en Bruselas, fue Gotthald Pannwitz y no el astrónomo Jean Mascart -como tienden a confundir algunos historiadores- quien organizó en marzo de 1910 la expedición científica que llegó a Las Cañadas para realizar diversas experiencias fisiológicas que están recogidas en el libro de Jean Mascart Impressions et observations dans un voyage à Ténérife, además de un seguimiento del cometa Halley en su aproximación al Sol.

Lo que vino a estudiar este grupo de fisiólogos europeos -los cuales permanecieron en Las Cañadas apenas 20 días, hasta el 18 de abril; Mascart se quedaría un mes más- fueron diferentes aspectos del organismo humano y su adaptación fisiológica a la altura, en unas condiciones climáticas más favorables en aquellas fechas que las reinantes en Davos, el Monte Rosa o el Mont Blanc, que era donde Zuntz y otros fisiólogos ya habían hecho estudios pioneros ${ }^{10}$. Pero esta elección estuvo determinada por el interés y el patrocinio de Gotthold Pannwitz y Hugo Hergesell, no porque ellos creyeran que estaban ante el mejor emplazamiento hasta entonces descubierto.

Los estudios y las opiniones de los diferentes doctores -Zuntz, Durig, Von Schrötter, Carriere y Douglas operaron en Altavista, mientras Mascart, Barcroft y Neuberg lo hacían en Guajara- hicieron famosas las palabras de Mascart: «La situación de Tenerife es quizás única en el mundo: es un faro, relativamente elevado, en medio del océano... Esta montańa [refiriéndose al monte del Teide] es, en efecto, particularmente favorable para las investigaciones de física y de astronomía». Por parte de los fisiólogos se concluyó que «las radiaciones solares de las Cañadas son de una magnitud única debido al aire puro exento de vapores y ser la luz solar siempre directa, de mejor efecto que la acción difusa».

Ambas conclusiones -que en nada aluden a la idoneidad del clima para combatir la tuberculosis ni las afecciones respiratorias- serán los principales argumentos que se esgrimirán durante décadas para defender el establecimiento de un sanatorio antituberculoso en el Teide.

\section{LAS CAÑADAS Y EL SANATORIO DEL TEIDE}

La presencia del observatorio alemán y de los científicos europeos en Las Cañadas del Teide -de amplia repercusión en la prensa local y nacional- dio automáticamente carta de naturaleza a las excelentes condiciones del lugar - La Cañada de La Grieta (foto 3) y su entorno- para el estudio del clima y la salud. De tal modo sucedió que nada más establecido el observatorio ya podían verse en torno al mismo numerosas personas que ya se alojaban en sus alrededores. El propio Mascart llegaría a quejarse a las autoridades espańolas del alojamiento en el observatorio meteorológico de algunos enfermos extranjeros enviados por el Humboldt Kurhaus ${ }^{11}$.

10 González Reimers, E. y Arnay de La Rosa (2010): La expedición científica de 1910 a Las Cañadas (Tenerife). Canarias médica y quirúrgica, mayo-agosto.

${ }^{11}$ De Ory Ajamil, F. (2019): Scientia Potentia Est. Las Cañadas del Teide, objetivo científico y geoestratégico del imperio alemán (1904-1916). Anuario de Estudios Atlánticos. 


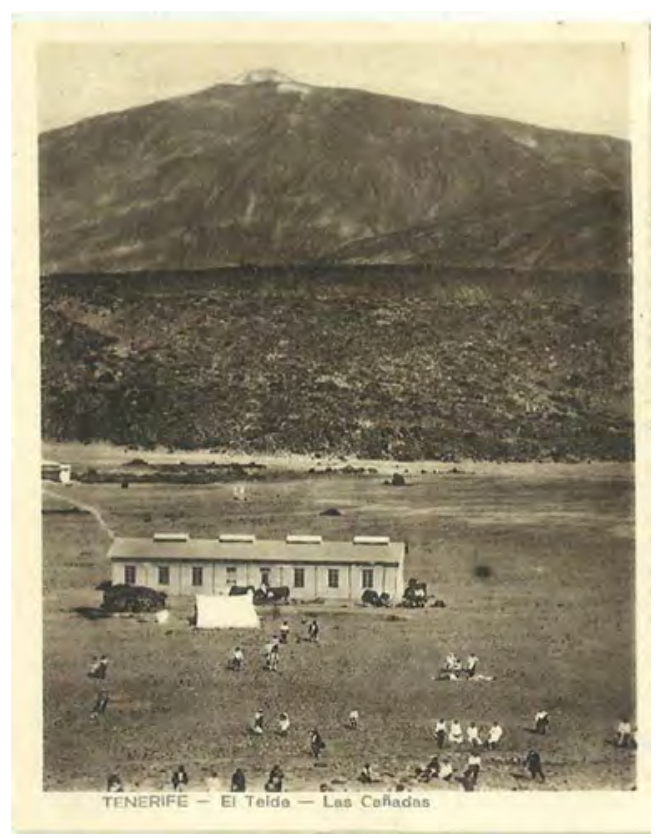

Fig. 3. Una treintena de personas alrededor del Observatorio de La Cañada de La Grieta. Circa 1910.

El conflicto político y diplomático entre España y Alemania por la presencia y actividades de sus científicos en Las Cañadas del Teide se resolvió con la construcción del observatorio meteorológico de Izaña, inaugurado el 1 de enero de 1916. Dos años antes había comenzado la primera guerra mundial y los científicos alemanes abandonaron aún antes el observatorio de La Cañada de La Grieta. El personal español se trasladó finalmente a Izaña, manteniendo en el primer observatorio algunos instrumentos y registradores meteorológicos hasta 1921.

Ante esta situación, muy pocos meses después, en una sesión municipal del ayuntamiento de La Orotava, de mayo de 1916,

leyóse un oficio del Sr. Subdelegado de medicina Inspector de Sanidad del Distrito, con fecha ocho del corriente, exponiendo las razones por las cuales interesa que esta Corporación solicite del Gobierno le conceda las casetas conocidas con el nombre de Casetas de los Alemanes que se hallan emplazadas en terrenos de este Municipio, donde llaman Las Cañadas, con objeto de utilizarlas como núcleo para establecer allí un Sanatorio de interés mundial, llamado a convertir a Tenerife en una gran centro de atracción universal y aprestar a la humanidad enferma y valetudinaria incontables servicios (...)

(...) Conforme con este parecer los demás Sres. Concejales, el Ayuntamiento acordó autorizar al Sr. Alcalde para que en nombre y representación del Consejo, eleve respetuosa instancia al Ministerio correspondiente, en súplica de que se digne 


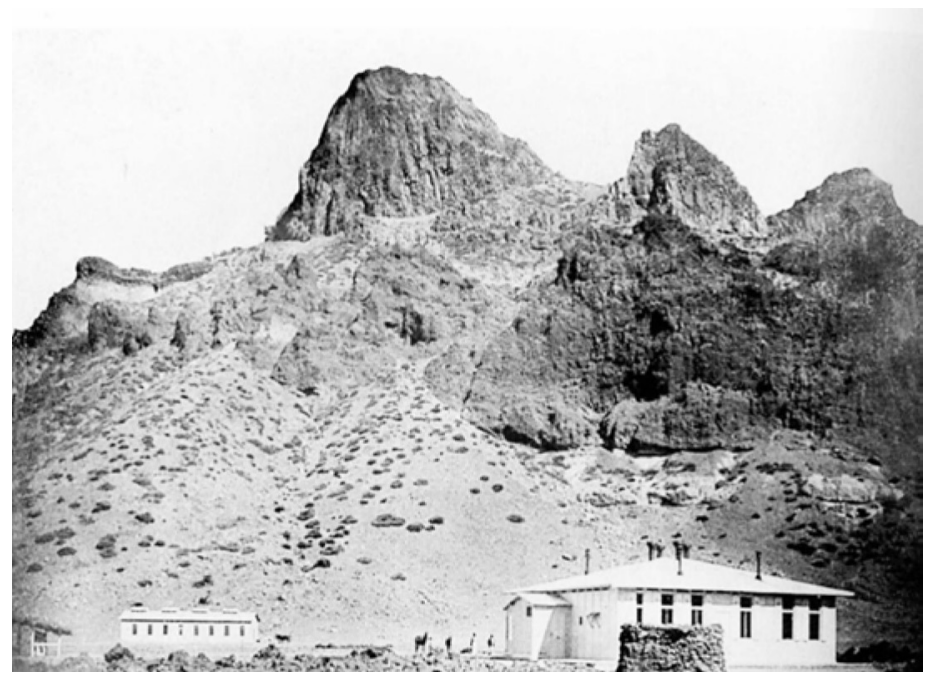

Fig. 4. Observatorio meteorológico alemán. La Cañada de La Grieta. Circa 1910.

conceder a la Municipalidad las casetas de que se ha hecho referencia, con el importante objeto que también queda indicado ${ }^{12}$.

Por parte del Ministerio de Instrucción Pública se denegó el uso y la cesión de tales "casetas", pues aunque eran de propiedad alemana acabaron reinstaladas tras el conflicto mundial en el Observatorio de Izaña, tras solicitar su venta a Espańa. Es por ello cuando aparece, a partir de entonces, un conjunto de iniciativas y reivindicaciones para movilizar recursos en torno a la construcción de un sanatorio antituberculoso en Las Cañadas del Teide.

Mientras tanto, para reclamar la construcción de un sanatorio antituberculoso en el Teide, se publicaron varios artículos en septiembre de 1918 exponiendo

muy atinadas razones acerca del enorme desarrollo que alcanza actualmente en Tenerife el terrible mal de la tuberculosis, cuyos estragos según estadísticas de los últimos meses llegan a una cifra verdaderamente aterradora (...). He de hacer notar las deficiencias que existen en Las Cañadas e inmediaciones del Teide, particularmente por la falta de albergues (...) las diez personas que se encontraban allí tuvieron que habitar en pequeñas chozas de piedra techadas de retamas haciendo una vida verdaderamente guanchinesca. Estas deficiencias es necesario subsanarlas en beneficio de los enfermos y por el propio decoro del país ${ }^{13}$.

12 Acta de la sesión municipal celebrada el 11.05.1916 en el Ayuntamiento de La Orotava.

13 La Prensa, 07.09.1918. 


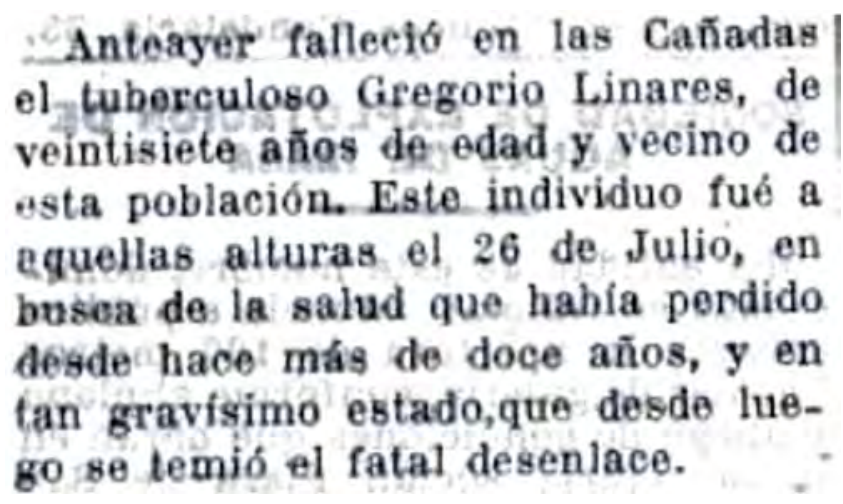

Recorte del diario La Prensa del 8 de agosto de 1919.

Un año más tarde, en junio de 1919, la prensa local citaba la llegada de «numerosos extranjeros atraídos por los maravillosos efectos del clima. Dos extranjeros, uno suizo y otro noruego, desahuciados por los médicos de los sanatorios europeos, han recobrado la salud perdida con sólo veinte días de vida en Las Cañadas»" ${ }^{14}$. También en agosto llegaron a Las Cañadas «más de 70 personas de La Orotava y Santa Úrsula», para pasar el verano en "construcciones de chozas de piedra cubiertas con planchas de zinc o, las más, con gajos de retamas $»^{15}$. Con relativa frecuencia-se añadía en la revista Las Canarias en aquel año- "los propios hijos de Tenerife y de las demás islas del Archipiélago en quienes ha hecho presa la funesta tuberculosis, emigran presurosos hacia países extranjeros, con preferencia a Suiza, ávidos de encontrar la curación de su padecimiento $»^{16}$.

En este sentido, un artículo del doctor Fernancruz comparaba Las Cañadas del Teide con «el sanatorio de Leysin (Suiza) que tiene cabida para seis mil enfermos y al que concurren, con la natural sorpresa por nuestra parte, algunos enfermos de estas Islas.....17.

En el verano de 1920, se afirmaba que fueron a Las Cañadas unos «ciento cincuenta enfermos, con el fin de recobrar la salud en aquellas saludables alturas. Por falta de viviendas construyen una especia de corrales o chozas de piedra pómez, allí tan abundante, y las cubren con ramas de retama blanca, especie vegetal que se da con abundancia en aquellas altitudes ${ }^{18}$.

\footnotetext{
${ }^{14}$ Las Canarias n. ${ }^{\circ} 1155,26.07 .1919$.

${ }_{15}$ Las Canarias n. ${ }^{\circ} 1156,11.08 .1919$.

${ }^{16}$ Las Canarias n. ${ }^{\circ} 1156,11.08 .1919$.

${ }_{17}$ Las Canarias n. ${ }^{\circ} 1158,11.09 .1919$.

${ }_{18}$ La Gaceta de Tenerife, 08.01.1921.
} 
En primer lugar, no es admisible, ni siquiera considerando ol caso eomo una eansecuencia de la iniciativa particular quo alli so establece como pudiera lacerlo en cualquier parte a su antojo, que exista una colonia enferma. instalada $\mathrm{e}$ sseis 0 más horas de poblado, por caminos pedregosos y prácticamente iutransilables. sin que haya un médice que la atienda y en cierto molo garintice at vida, a la vez quo contribuya a la curación de muchos de aqueltos intelices con la confian2a. $y$ la fe que logre inspirarles.
El climá, el aire, la radiación de la. Juz, In influoncía benéfiea de los rayos uliravioleta, todo eso del más sano y puro benefieio orgínieo, es cierlaments el factor principelisimo paca la curacion en las Cáadas; pero de igual modo puede ser fatal en aquellos cesos en que, por la ausencis de un medico, el enfermo se autorroceta, dándoso baĩos do sol 0 de aire, 0 , simplemente, respirando allí dondo sus pulmones no encuentran yo medios para soportar la endesa de ciertos reactivos.

Recorte del diario La Prensa, del 27 de agosto de 1919.

Los riesgos a los que se exponían los enfermos que subían a Las Cañadas del Teide llevaron a los periódicos de la época a actuar a modo de emisarios:

Los enfermos deben consultar con su médico antes de emprender la marcha a aquella región. Demuestra también este caso [el recogido en el recorte adjunto] la necesidad imperiosa del sanatorio para menesterosos (...) demuestra, por último, la urgencia de que un médico se establezca allí para prestar sus auxilios a aquellos enfermos, que sin dirección facultativa, están expuestos a sufrir fatales consecuencias. Acerca de este importante asunto, llamamos la atención de la Junta de Fomento de Las Cañadas que acaba de crearse ${ }^{19}$.

El panorama encontrado en las cumbres durante estos años debió ser, cuando menos, caótico. Hacia 1919, afirmaba un periódico local:

Cuando la afluencia de enfermos deseosos de disfrutar de aquel clima fue extraordinaria, se pudo observar la existencia de chozas y corrales que habían construido los pastores. Entonces se restringió algo - no en absoluto- el pastoreo. Pero, más tarde, en julio de 1926 se sorprendieron unas 152 cabezas de ganado, a cuyos dueños se les impuso la multa correspondiente. También en 1928 hubo otra denuncia, que ha recorrido por artes mágicas todos los trámites y peripecias de las dilaciones. Figuraba como delincuente nada menos que el que era en aquellos días alcalde del pueblo de Granadilla. y en 17 de febrero del mismo año se ordenó que se suspendiera la exención de la multa impuesta, hasta que el Juzgado resolviera sobre un sumario ${ }^{20}$.

19 La Prensa, 08.09.1918.

20 Las Noticias, 17.08.1929. 

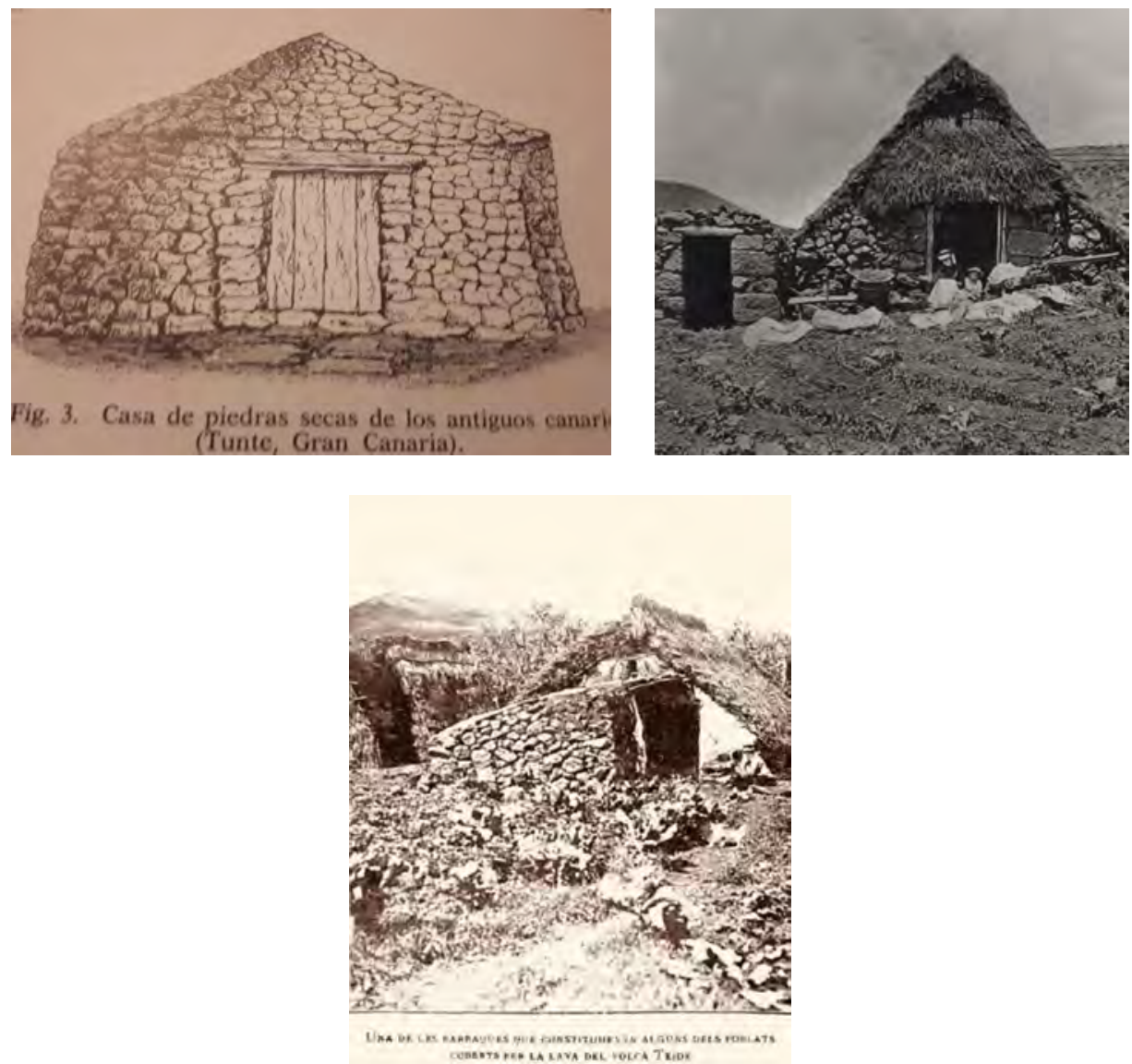

Fig. 5. Diferentes tipos de chozas o corrales en las islas Canarias.

El mismo diario Las Noticias aseguraba que en el verano de 1929 pastaban por Las Cañadas del Teide más de tres mil cabras ${ }^{21}$ y otra fuente asegura que a fines de la década de 1920 se calculaba que existían entre cinco y seis mil cabras en Las Cañadas y zonas limítrofes, rebaños que pastaban sin vigilancia, como era la costumbre en la isla ${ }^{22}$.

Un artículo escrito por el político portuense Luis Rodríguez Figueroa - bajo el pseudónimo Guillón Barrús- publicado en el diario La Prensa en julio de 1924

${ }^{21}$ Las Noticias, 02 y 03.08.1929.

22 García Mesa, Domingo A., Núñez Pestano, Juan Ramón y Quirantes González, Francisco (2015): «La lucha por la apropiación de los recursos y el fin de los aprovechamientos comunales en las Cañadas del Teide». Revista de Historia Canaria n. ${ }^{\circ}$ 197, p. 132. 

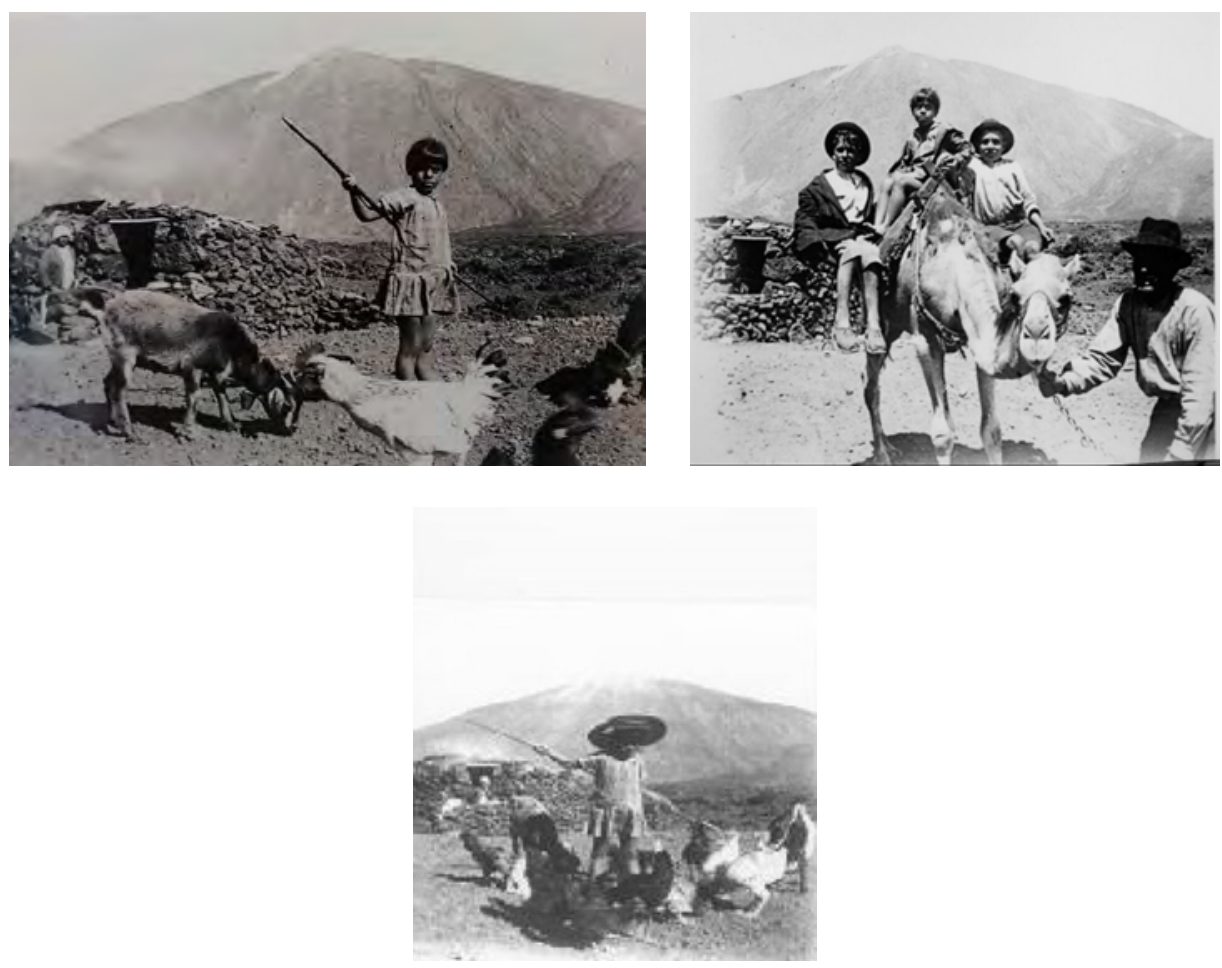

Figs. 6, 7 y 8. Imágenes de un corral habitado en Las Cañadas del Teide durante el verano de 1925.

es revelador de varios puntos de interés sobre el asentamiento de enfermos en La Cañada de La Grieta:

Lo que vi al llegar a las casetas de los alemanes, en la Cañada de la Grieta, donde nace el manantial de este nombre, me dejó estupefacto. Acampados en el gran perímetro de aquel paraje viven temporalmente, como Dios quiere, en chozas de piedra seca, mal cubiertas por ramajes de retamas, unos cuarenta y tres enfermos, algunos $-\mathrm{y}$ no quiero hacer una frase de ocasión, que pudiera ser de cruel impertinencia si no fuese exacta- en tal estado de aniquilamiento, que yo, en el primer instante, solo pude explicarme que les hayan subido hasta allí para hacerles más corto el viaje a la Eternidad. Así están los infelices de maltrechos, de acabados por el terrible mal de la tisis.

Otro aspecto de enorme interés, comentado por esta personalidad local, se refiere al «aspecto social de la tuberculosis: declaro en este sentido que aquí, en Canarias, va siendo aterrador el problema, por incuria higiénica, por insuficiencia nutritiva, por falta de celo en la inspección de las substancias alimenticias, por el descuido más absoluto con que se mira la educación física... ¡qué se yo!... por una infinidad de circunstancias que desatienden las autoridades locales, y los padres, y los inspectores 
de enseñanza y, en general, la masa consciente de la población, donde no se hace más que vivir al día, llenos de vanidades pueblerinas...».

Por lo que se refiere al estado de conservación de las «casetas de los alemanes», el citado político comenta:

Con asombro reconcentrado recorrí los diversos compartimentos de una construcción que el transcurso de cinco o seis años ha sido local «ad hoc» para una serie de experiencias de diversa índole, y taller donde altas personalidades de la Ciencia se han afanado por aportar factores últimos al bienestar humano. ¡Y qué vergüenza!, he visto destrozadas las grandes planchas de amianto de la caseta, ahumada, ennegrecida su techumbre, como la de una cueva de facinerosos; rebosando de excrementos cabrío y caballar el piso, y amontonada hasta la altura de un metro la ceniza de la candela con la que por largo tiempo se ha venido haciendo de comer en un ángulo convertido en fogón.

En el verano de 1924, la prensa recogía que «en esta época del año, Las Cañadas, en donde moran muchísimas personas en busca de salud -actualmente rebasan la cifra de un centenar de ellas-, ofrecen el aspecto de una aldea mora o india; quizás "las chozas" que cobijan a los enfermos semejen las viviendas de los guanches, la raza primitiva, noble y vigorosa (...) es muy importante hacer notar que las personas que han acudido y acuden a las Cañadas en busca de salud, no son tuberculosos solamente; las hay que padecen del estómago, reumáticas; sobre todo, las que sufren úlceras exteriores de cualquier clase, obtienen su total curación a los pocos días» ${ }^{23}$.

\section{LA JUNTA DE FOMENTO DE LAS CAÑADAS DEL TEIDE. LA CONSTRUCCIÓN DEL SANATORIO}

En la creación de esta Junta confluyeron diferentes intereses y, entre ellos, el principal fue la construcción de una carretera que, atravesando Las Cañadas del Teide, uniera las poblaciones de La Orotava y Vilaflor. Ello dio lugar a no pocos debates y protestas, fundamentalmente del municipio sureño, sobre el trazado de la misma. Además de la promoción de Las Cañadas para la instalación de un sanatorio para tuberculosos, dicha Junta planteó la creación del Parque Nacional del Teide -ya solicitado en 1917-, la instalación de un «funicular», la construcción de un hotelalbergue, la repoblación forestal, y todo ello dirigido hacia la explotación del incipiente turismo que venía desarrollándose en la isla y quería trasladarse a sus cumbres.

Así, el 4 de enero de 1920 el diputado a Cortes Julián Van Baumberghen, que a la sazón era inspector provincial de Sanidad, marchaba a Madrid «llevando toda la documentación, antecedentes, resumen de los trabajos, etc., a fin de some-

${ }^{23}$ La Prensa, 02.08.1924. 


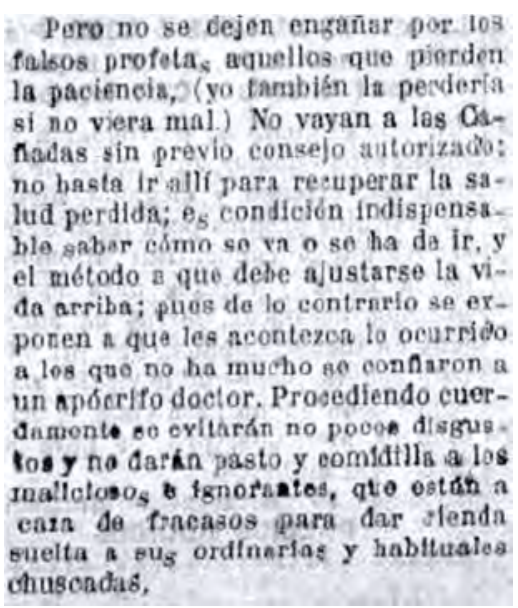

Recorte del diario La Prensa, 17.10.1922.

terlos a la Superioridad, y recabar que, con la mayor urgencia posible, comience el envío de cantidades para los trabajos preliminares de instalación $»^{24}$.

Por lo que se refiere a la construcción del sanatorio, el 20 de junio de 1921 se publica en el Boletín Oficial de la Provincia la subasta pública por importe de 237 274,50 pesetas para la construcción de las obras de conducción de aguas al lugar donde ha de emplazarse el Sanatorio del Teide, con arreglo al pliego de condiciones que también se inserta ${ }^{25}$. Un año después, el 9 de junio de 1922 , se cita que la representación parlamentaria tinerfeńa, cerca de la Comisión de Presupuestos del Estado, "ha dado por resultado halagüeńo el que se incluya la cantidad de 250000 pesetas para la construcción del Sanatorio de Las Cañadas del Teide» ${ }^{26}$. Y en octubre del mismo año se adjudican las obras del mismo a Roque Montesdeoca -el mismo contratista que construyó el Observatorio de Izaña- por importe de 225733 pese$\operatorname{tas}^{27}$, siendo proyectada por Antonio Pintor Ocete, arquitecto municipal de Santa Cruz de Tenerife.

Los problemas que encontró el constructor del Sanatorio fueron prácticamente los mismos a los que se enfrentó diez ańos antes durante la construcción del observatorio meteorológico de Izaña: la imposibilidad de trabajar durante seis u ocho de los doce meses por el clima, la inexistencia de una carretera que facilitara el acarreo de materiales y el coste de mano de obra desplazada hasta las cumbres.

24 La Prensa, 31.12.1920.

25 BOP, 20.06.1921.

${ }^{26}$ La Gaceta de Tenerife, 9.06.1922.

27 Heraldo de La Orotava, 22.10.1922. 

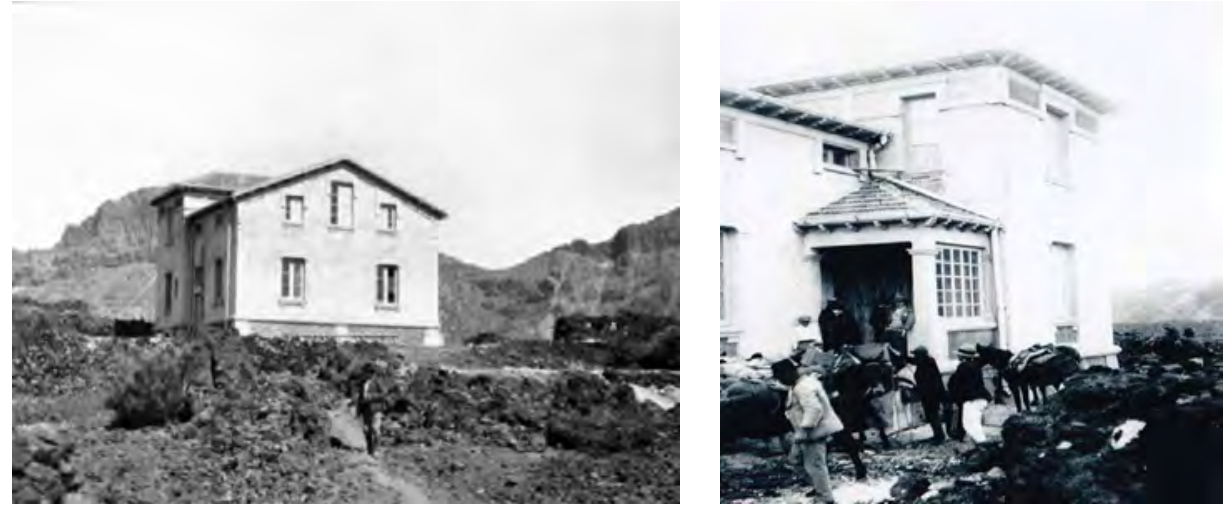

Figs. 9 y 10. «Casa del Médico», año 1925.

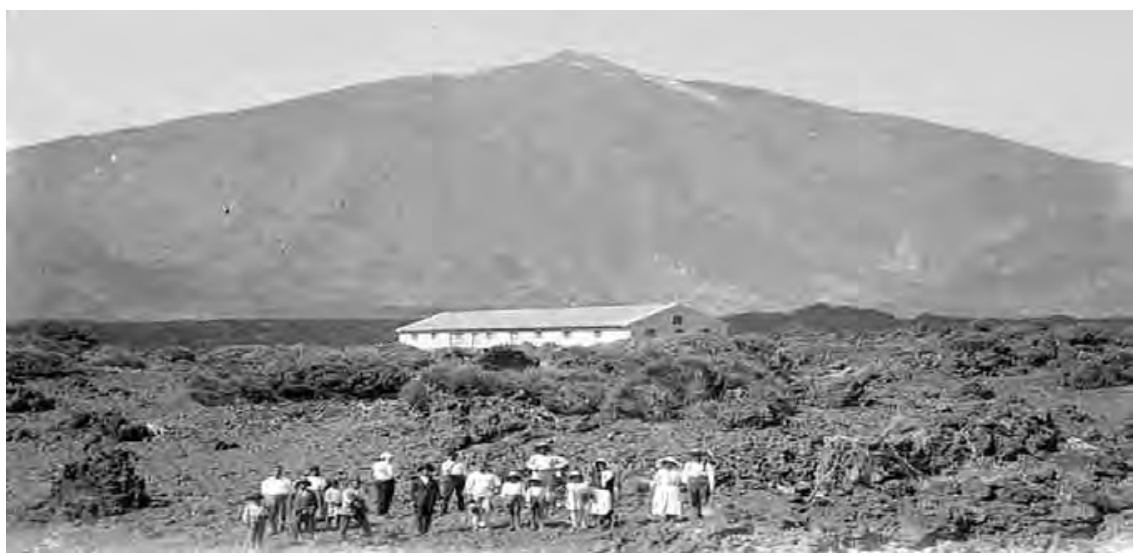

Figs. 11 y 12. El garaje o "Gañanías», años 1940 y 1960.

Sin embargo, en agosto de 1923 se publica que «personas llegadas en estos días de "Las Cañadas", nos dicen que están al terminar las obras del primer grupo del Sanatorio del Teide, cuyos trabajos dirige el maestro de obras don Roque Montesdeoca. Dichas personas hacen grandes elogios de la belleza y solidez de los distintos departamentos construidos, así como de la instalación de aguas del referido edificio» ${ }^{28}$. Lo cierto es que en abril de 1925 el chalet del Sanatorio estaba definitivamente construido, pues dio alojamiento a varios doctores extranjeros, tal y como se publicaba en la prensa local.

28 La Prensa, 04.08.1923. 


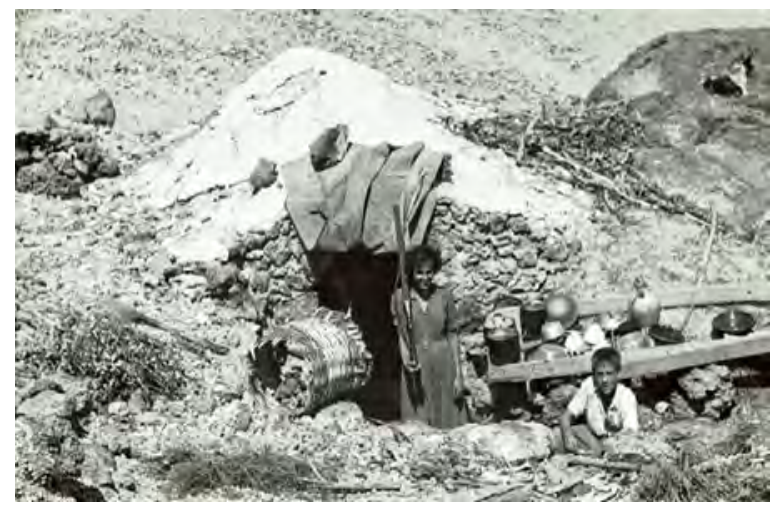

Fig. 13. Corral en la cara Sur del Teide. Circa 1950.

\section{SOBRE LA IDONEIDAD DEL SANATORIO DEL TEIDE. DEBATES ENTRE ANTI- Y PRO CAÑADISTAS. LA OROTAVA VERSUS VILAFLOR}

A modo de presentación y síntesis traemos a colación de esta controversia el parecer de dos autores anónimos que publicaron frecuentemente artículos en contra y a favor de la existencia de un Sanatorio en las cumbres. Así, pues,

habrán visto nuestros lectores que Gaceta de Tenerife, solícita y atenta, ha publicado estos días interesantes artículos de «El Conde de las Veguetas» y «Juan de Izaña» respecto a las condiciones climatológicas de nuestras Cañadas para establecer un Sanatorio para tuberculosos. «El Conde de las Veguetas» pone en duda los efectos benéficos de Las Cañadas para tan cruel enfermedad. «Juan de Izaña» cree, por el contrario, que son inmejorables (...) la opinión está bastante dividida, pues mientras que unos opinan que un Sanatorio para tuberculosos en las Cañadas sería, a más de una obra humanitaria, un venero de riqueza para Tenerife, y llevaría el nombre de nuestra querida isla por el mundo entero, creen otros que sin negar el humanitarismo, meramente altruista en lo que se refiere a nosotros, pero ineficaz en lo que se refiere a los enfermos, está muy lejos de ser venero de riqueza, ahuyentaría el turismo saludable y encierra un peligro muy serio para los habitantes de Tenerife ${ }^{29}$.

Aparece entonces en escena el doctor Tomás Zerolo Herrera, entre otros muchos, abogando por Vilaflor como estación más idónea que Las Cañadas para el establecimiento de enfermos. Además de su más adecuada altitud -la cita a 1600 metros sobre el nivel del mar-, «su atmósfera, saturada por las saludables emanaciones resino balsánicas de aquellos árboles, es seca como ninguna, fuertemente tónica y tan pura y antiséptica que las materias orgánicas conservan tenazmente su vitali- 


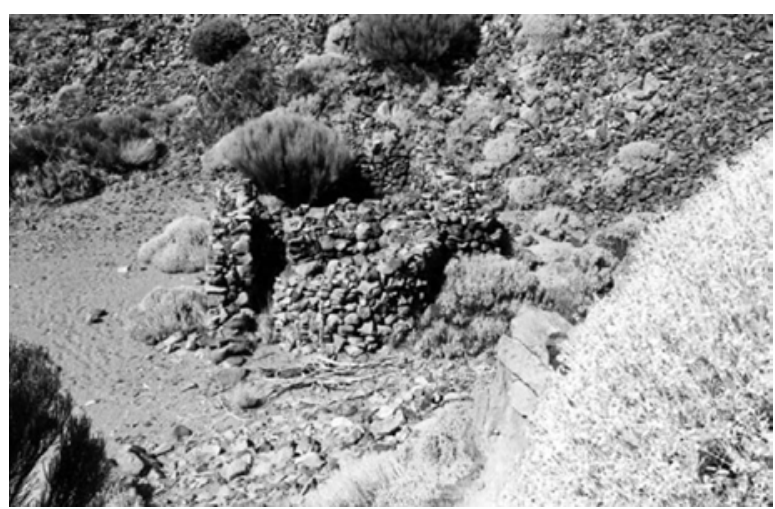

Fig. 14. Resto de choza de fecha reciente en La Cañada de La Grieta.

dad y casi no entran en descomposición. Posee Vilaflor, además, manantiales de agua minero-medicinales, principalmente indicadas en las afecciones de las vías digestivas ${ }^{30}$. Igualmente relevante merece ser el criterio del doctor Tomás Cerviá Cabrera -quien fuera el promotor y fundador del Hospital Antituberculoso de Santa Cruz de Tenerife-. En un artículo publicado en La Prensa el 31 de julio de 1928, afirmaba rotundamente que

sobre las mejores ventajas del clima de las Cañadas o Vilaflor para el tratamiento de la tuberculosis pulmonar y el emplazamiento de un Sanatorio para esta clase de enfermos, nos creemos en el deber de exponer ante la opinión pública interesada en estas cuestiones, porqué elegimos Vilaflor como lugar más indicado para la construcción de un Sanatorio de altura que en breve emplazaremos alli ${ }^{31}$. Antes que nada debemos declarar que no somos del Sur ni del Norte de esta isla -Cerviá era natural de la isla de La Palma-, que no tenemos interés alguno en dichas regiones y que creemos haber estudiado el tema desapasionadamente, de acuerdo con el estado actual de la Ciencia, pues en ello van nuestros intereses. Buscando el lugar para la erección del Sanatorio eliminamos enseguida las Cañadas, sin haber estado en ellas, por el simple dato de su excesiva altura, pues serían muy pocos los pacientes que la pudieran soportar sin graves perturbaciones. Si a esto unimos la completa despoblación forestal, condición que consideramos indispensable, nuestra decisión en este sentido fue inmediata. Estuvimos en Vilaflor y nos convenció inmediatamente por su altura $(1366 \mathrm{~m})$ análoga a la de los más acreditados sanatorios de fama mundial; por sus pinos, ricos en emanaciones balsámicas; por su fácil acceso desde la carretera, (no

${ }^{30}$ Heraldo de La Orotava, 04.02.1923.

31 En el pueblo sureño de Vilaflor de Chasna, el 12 de marzo de 1928, varios médicos adquirieron un terreno de considerables dimensiones (siete hectáreas, 65 áreas y nueve centiáreas), en una zona llamada el Carrillo, por 300 pesetas. Según figura en la escritura de compra-venta, el solar fue adquirido por Antonio Pérez Díaz, Tomás Cerviá Cabrera, Tomás Zerolo Fuentes y Juan Friend Martín, para la construcción de un sanatorio antituberculoso, establecimiento que nunca vio la luz. 
antes habrá Sanatorio); por su buena orientación, y por lo abrigado que podría estar de los vientos (...). A pesar de todo nos interesaba conocer las Cañadas - -tanto se habla de ellas!- y, recientemente, aprovechando la invitación de unos amigos, estuvimos allí. No ignorábamos los trabajos que las diferentes comisiones científicas habían efectuado en ellas, y sabíamos de las riquezas en radiaciones actínicas y en conductibilidad eléctrica que dichos investigadores comprobaron, y que nosotros gracias a un magnífico eritema solar que adquirimos, por experiencia propia, también pudimos comprobar. Igualmente comprobamos que nuestro miocardio - de hombre sano pero poco acostumbrado a ascensiones tan molestas- se fatigaba. Y pensábamos $\mathrm{Y}$ ya en plenas Cañadas, donde vimos las célebres chozas y aquel principio de Sanatorio ya abandonado, nos decíamos: ¿Es posible que un ser humano, enfermo, se cobije bajo una choza -cuatro piedras y un techo de retamas-y sufra el rigor de un Sol como el que soportamos, con los peligros que para él como enfermo del pecho representa? (...). ¿Es posible que un enfermo sin conocimiento del método de tratamiento que más le conviene, carezca de la vigilancia de un médico que le dirija y viva sin los cuidados de alimentación y medicamentos necesarios para su precario estado de salud?...

\section{FRACASOS DE LA JUNTA Y LA GESTORA DE LA LUCHA ANTITUBERCULOSA}

El 1 de abril de 1926 se constituyó en Santa Cruz de Tenerife la Junta Provincial de la Lucha Antituberculosa, creándose el Dispensario General Primo de Rivera. Sin embargo, en sucesivos años se denunció públicamente su fracaso y abandono por parte de las autoridades, llegando a afirmarse que «en materia de tuberculosis, nuestra sociedad -lo mismo las clases superiores que las populares- viven con un siglo de atraso» ${ }^{32}$.

Mientras tanto, en abril de 1927, la prensa continuaba ocupándose del abandono en el que permanecían los enfermos que acudían a Las Cañadas del Teide «en busca de alivio para sus padecimientos». A tal efecto citaba dos casos angustiosos ocurridos en aquellos solitarios parajes:

Un obrero -doliente de los pulmones-, ya desahuciado por la Ciencia sin contar con otros recursos que la caridad y la protección exigüa de un hermano, se encaminó, en último recurso, a las Cańadas en compañía de su madre. En lo alto de un pedregal, expuesto a los cuatro vientos, le levantaron su choza (...) a los pocos días le faltaba el aire; no podía inspirarlo. Solo, sin lenitivo medicinal alguno, a brazo partido con la muerte, sucumbió en medio de los espasmos más atroces (...) el cadáver fue llevado al pueblo a cuestas de una mula (...) un extranjero también ansioso de salud, sin norma ni consejo a que sujetarse para lograr su cura, se debilitaba, perdía por momentos sus fuerzas. Cuando, después de tantas alternativas de su enfermedad, comprendió que allí no había para él remedio, optó por marcharse. A lomos de una mula iba sujeto en unas angarillas por un amigo. Apenas había

${ }^{32}$ La Gaceta de Tenerife, 25.01.1930 
avanzado, cuando un sofoco le obligó a apearse. Sintió fatiga; se desvaneció. Los gritos llorosos de su esposa anunciaron su muerte al infinito (...) del proyectado sanatorio de las Cañadas existen actualmente dos pabellones construidos: uno destinado para casa del médico; otro, para garage. Aprovechando esta circunstancia ¿no se podría gestionar, mediante la consignación que correspondiera, que por lo menos, un médico residiese en las Cañadas durante la época en que los enfermos acostumbran acudir a aquellos lugares? ${ }^{33}$.

En marzo de 1932 se comunicaba que

mañana se cumple el medio año que lleva de constituida en esta capital la Comisión Gestora de la Lucha Antituberculosa (...) que el actual abandono con que se viene caracterizando la obra efectiva de dicho organismo (...) se va agravando en Tenerife la propagación tuberculosa, en términos que ya pone a nuestro país en alarma inquietante y justificada. Nada hay que explique satisfactoriamente esta especie de enfermedad del sueño [sic] de que parece estar acometida la mencionada Comisión Gestora ${ }^{34}$.

En mayo de 1933 se advertía de la desaparición de la Junta Provincial y que a finales de abril de ese año se había inaugurado en Santa Cruz un Dispensario Antituberculoso «dotado con los perfeccionamientos científicos que esa clase de Establecimientos requieren y dirigido por el joven doctor don Tomás Cerviá Cabrera, reputado especialista en tisiología» ${ }^{35}$. Pero en el mismo artículo el doctor F. Jerez Veguero -director del Hospital de La Orotava-, consagrado al problema de la tuberculosis, señalaba que

Tenerife carece hoy día de lo más elemental, de lo más humano en cuestiones de este orden sanitario: de una Enfermería Antituberculosa. Esto es una gran verdad. Como lo es, también, que esa Enfermería no se encuentra actualmente funcionando en Tenerife por haber cesado en sus funciones la Junta Provincial de la Lucha Antituberculosa a que hemos aludido. ¡Una vez más, el entrometimiento de la política vino a malograr una obra altamente beneficiosa para la isla entera ${ }^{36}$.

\section{EL FINAL DEL SANATORIO DEL TEIDE Y LA CREACIÓN DEL HOSPITAL ANTITUBERCULOSO DE SANTA CRUZ DE TENERIFE}

El 24 de diciembre de 1933 el diario La Prensa de Santa Cruz de Tenerife publicaba una entrevista con el doctor Juan Torres Gost -quien poco después sería nombrado director del Hospital Nacional de Enfermedades Infecciosas-, quien

\footnotetext{
33 El Progreso, 02.04.1927

34 La Gaceta de Tenerife, 02.03.1932.

35 La Gaceta de Tenerife, 28.05.1933.

36 La Gaceta de Tenerife, 28.05.1933.
} 


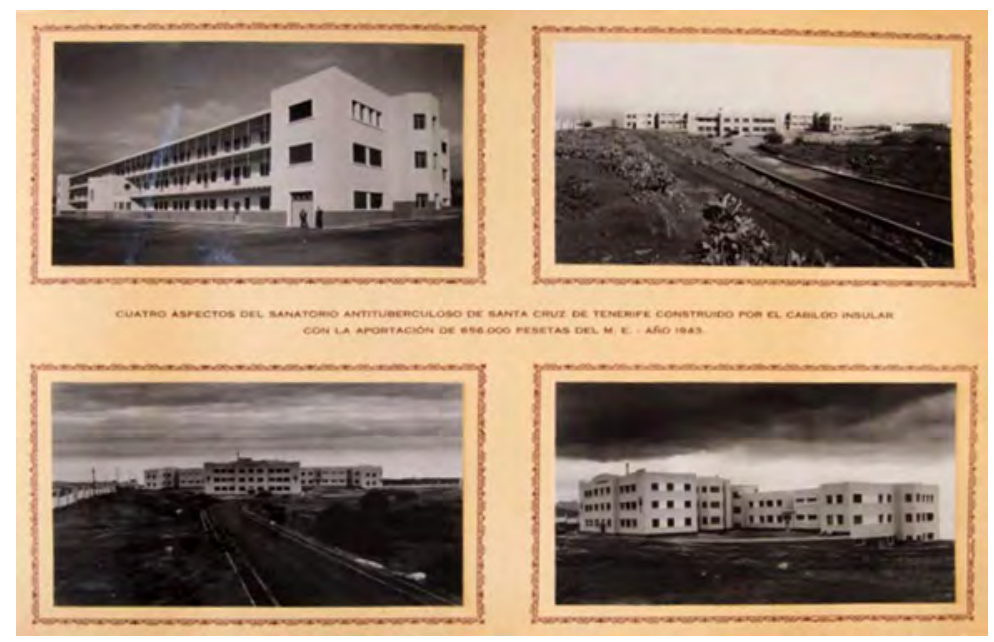

Fig. 15. Construcción del Hospital Antituberculoso de Santa Cruz (hoy Hospital Universitario de Canarias). Año 1943.

se declara decidido partidario, por diversas razones, de un Sanatorio o Enfermería Antituberculosa en esta capital (...) desechando por el momento los Sanatorios de Las Cañadas o Vilaflor, que pueden quedar relegados a la iniciativa particular, puesto que por el coste de su sostenimiento, sólo podrían ser utilizados por enfermos ricos, y los Sanatorios oficiales deben estar al alcance de todo el mundo.

\section{Continuaba el diario señalando que}

la respetable opinión y los consejos del doctor Torres Gost, parecen haber tenido buena acogida en una reunión celebrada en el Cabildo, y acerca de ésto nada nos corresponde manifestar a los legos en la materia. La ciencia recomendó la construcción del Sanatorio de Las Cañadas, como lugar privilegiado para ello, y también la ciencia recomienda ahora el que se emplace en esta capital, como de resultados más prácticos y beneficiosos, especialmente para los pobres que son los que más lo necesitan...

En el año 1934 se declaraba como una obra abandonada el Sanatorio de Las Cańadas ${ }^{37}$ pero, en cambio, dicho diario volvía a citar que

actualmente pernocta en las Cañadas un centenar de personas que buscan su salud en aquellos apartados lugares, viviendo en chozas construidas con piedras y ramajes, sin poder disfrutar por más de tres meses escasos del balsámico clima y haciéndose cundir la especie de que su instalación ofrecería serios peligros para la salud de los

37 La Prensa, 14.08.1934. 
habitantes de Tenerife, sin embargo de que se recomiende después de la fundación de un Sanatorio Antituberculoso en la capital de la isla.

En una ascensión llevada a cabo por unos ingenieros catalanes -Fenech y Talens- con el presidente del Cabildo, entonces Maximino Cea, para elaborar un estudio preliminar para la construcción de un funicular en el Teide y que fue recogida por el periódico La Prensa el 19 de noviembre de 1935, se señalaba:

¡El Sanatorio! El Sanatorio que pudo ser. Que se quiso que fuera. Y que no ha sido nunca. Se hizo la casa del médico... Y «el garaje». Los muros sirven hoy de refugio a los excursionistas que por esta parte de Las Cañadas llegan, y de albergue, en la noche, a las gentes de las bandas del Sur que llegan a las tierras altas en busca de retama para hacer carbón. Recuas interminables cruzan estas laderas cargadas de carbón y leńa sacados de las cada día menos abundantes retamas del Teide. Esta gente es la que suele frecuentar el Sanatorio.

Así pues, una vez desechada la idea de proseguir con la construcción del Sanatorio del Teide, cobró importancia la creación del Hospital Antituberculoso de Santa Cruz. La presencia del doctor Tomás Cerviá Cabrera resultó de capital importancia en la fundación del Hospital Antituberculoso de Tenerife. En el primer Dispensario Primo de Rivera se hallaba el doctor Cerviá en octubre de 1928, pasando a dirigirlo desde 1932. En 1937 se encargó al Cabildo la construcción del nuevo Sanatorio-Enfermería Antituberculoso de Ofra. En aquel mismo año «basta decir que la tuberculosis causa en nuestra provincia unas 250 víctimas anuales; son 250 vidas, en su mayoría jóvenes, y de todas las clases sociales, que se pierden estérilmente» ${ }^{38}$. Pero no sería hasta el 8 de agosto de 1944 cuando los 96 enfermos que estaban ingresados en «El Palomo» fueron trasladados al nuevo Sanatorio, que si inicialmente, tenía 125 camas, fue proyectado para 225 pacientes.

Todavía en el año 1950 el doctor Cerviá afirmaba que «ningún problema médico sanitario tiene hoy la envergadura de la tuberculosis (...) la elevada mortalidad por esta causa (unas 20000 anuales en nuestro país, y no es de las más elevadas de Europa) no es superada por ninguna otra enfermedad.... ${ }^{39}$. Una detenida lectura de sus Trabajos del Sanatorio Antituberculoso de Ofra y del Dispensario Central-redactados en el año 1952- revela que aún entonces el problema de la tuberculosis de la isla estaba muy lejos de eliminarse y resolverse.

38 La Prensa, 15.08.1937.

39 «El Día Anual de la Tuberculosis», publicado en la Revista Española de la Tuberculosis 19/4 (227-281), abril 1950 . 


\begin{tabular}{|c|c|c|c|c|c|c|c|c|c|c|}
\hline \multicolumn{11}{|c|}{$\begin{array}{l}\text { CUADRO I } \\
\text { ANOO } 1950\end{array}$} \\
\hline \multirow[b]{2}{*}{ Meses } & \multicolumn{4}{|c|}{ Personas vistas por $10^{*}$ vez } & \multirow{2}{*}{\multicolumn{2}{|c|}{ 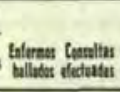 }} & \multirow[b]{2}{*}{$\begin{array}{l}\text { Butlonflus } \\
\text { Bdiousiat }\end{array}$} & \multirow[b]{2}{*}{$\begin{array}{l}\text { foles: } \\
\text { copiar }\end{array}$} & \multirow{2}{*}{\multicolumn{2}{|c|}{$\begin{array}{c}\text { Tuberedina } \\
+-\end{array}$}} \\
\hline & v. & H. & N. & Totales & & & & & & \\
\hline Enero ............... & 88 & 94 & 54 & 236 & 39 & 1.230 & 795 & - & 214 & 74 \\
\hline Febrero ............. & 64 & 107 & 54 & 225 & 31 & 1.296 & 751 & - & 216 & 80 \\
\hline Marzo . . & 93 & 127 & 75 & 296 & 52 & 1.733 & 828 & 194 & 320 & 107 \\
\hline Abril ................ & 78 & 114 & 62 & 254 & 23 & 1.230 & 660 & 166 & 179 & 79 \\
\hline Mayo ................ & 90 & 96 & 74 & 260 & 52 & 1.411 & 698 & 140 & 76 & 35 \\
\hline 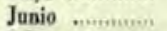 & 126 & 141 & 116 & 383 & 44 & 1.460 & 1.252 & - & 200 & 35 \\
\hline Julio .............. & 84 & 116 & 103 & 303 & 41 & 1.261 & 741 & 102 & 160 & 82 \\
\hline Agoste ............ & 89 & 129 & 112 & 330 & 46 & 1.270 & 917 & 120 & 183 & 61 \\
\hline Septiembre ....... & 83 & 108 & 70 & 261 & 33 & 1.039 & 666 & 360 & 161 & 34 \\
\hline Oetubre & 140 & 141 & 101 & 382 & 71 & 1.670 & 1.095 & 150 & 227 & 45 \\
\hline Noviembre ,...... & 69 & 77 & 98 & 244 & 36 & 1.223 & 153 & - & 171 & 29 \\
\hline Dieiembre & 61 & 83 & 70 & 214 & 73 & 1.215 & 545 & - & 227 & 21 \\
\hline Totales .......... & 1.065 & 1.334 & 989 & 3.388 & 541 & 16.038 & 9.011 & 1.232 & 2.334 & 685 \\
\hline
\end{tabular}

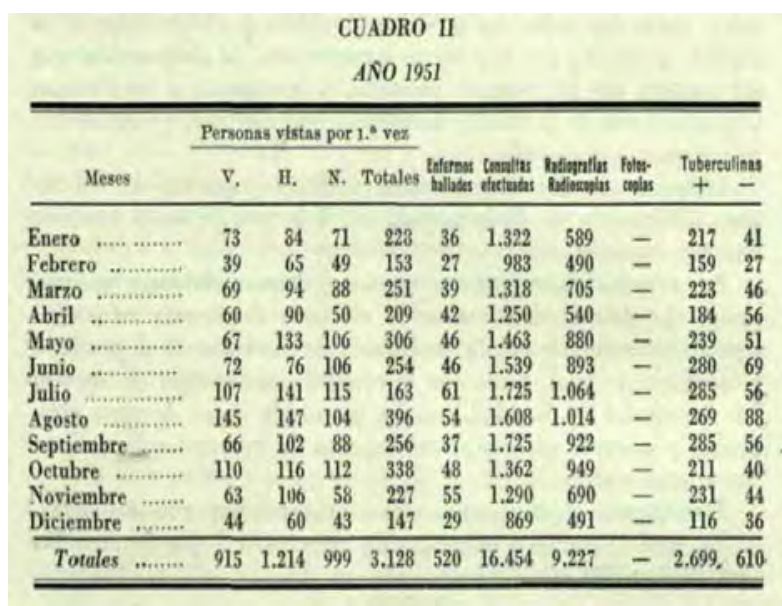

Cuadros I y II. Estudio pormenorizado de enfermos tuberculosos en la isla de Tenerife. Años 1950-1951.

\section{LA CREACIÓN DEL PARQUE NACIONAL DEL TEIDE Y LA APARICIÓN DE NUEVAS CONTRUCCIONES EN EL ENTORNO DEL SANATORIO}

La creación del Parque Nacional del Teide a partir de un real decreto de 22 de enero de 1954 del Ministerio de Agricultura -37 años después de la solicitud hecha al Estado por el concejal del Ayuntamiento de La Orotava Juan Acosta Rodríguez- modifica «nominalmente» el entorno del Teide y Las Cañadas. Como señala con amplitud Tomás Pérez Méndez: 


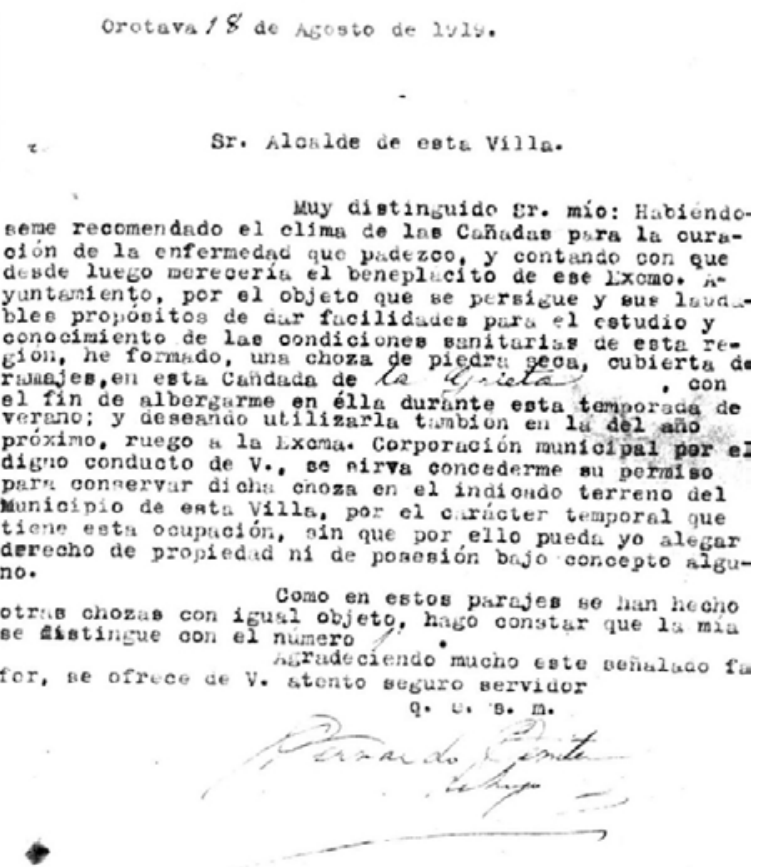

Carta autógrafa de Bernardo Benítez de Lugo solicitando permiso al Ayuntamiento de La Orotava para la construcción de una choza (la «número 1»).

La primera cuestión que se plantea es la prohibición dentro de su perímetro de la actividad minera. Pero, a pesar de ello, siguieron las extracciones de piedra pómez hasta comienzos de la década de 1980. Igualmente, se realizaron extracciones de rocas y piedras volcánicas en Las Cañadas, pues hasta la década de 1970 la administración del Parque Nacional autorizó unas 300 peticiones, la mayoría de ellas de empresarios de la construcción. A este volumen se unió el de las fincas privadas, principalmente de la finca Cumbres de Arico, de los herederos de Martín Rodríguez, donde se realizó la extracción de áridos (arena y toquilla volcánica) ${ }^{40}$.

El antiguo territorio comunal de Las Cañadas del Teide pasó a ser un nuevo espacio, «legalmente» natural y protegido. De modo que, a mediados de 1954, cuando ya se había creado el Parque Nacional, se procedió a recalificar el carácter comunal de las fincas denominadas Las Cumbres y El Teide, pertenecientes al Ayuntamiento

40 Pérez Méndez, Tomás (2000): Antecedentes históricos del Teide y Las Cañadas del Teide. La Orotava, pp. 62 y 72. La Orotava. 


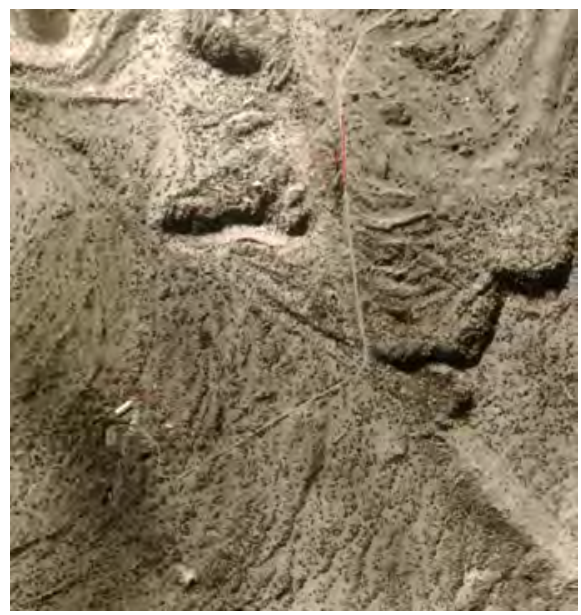

Fig. 16. Vista aérea de 1956: la Casa del Médico y Las Gañanías. Hoy desaparecidas.

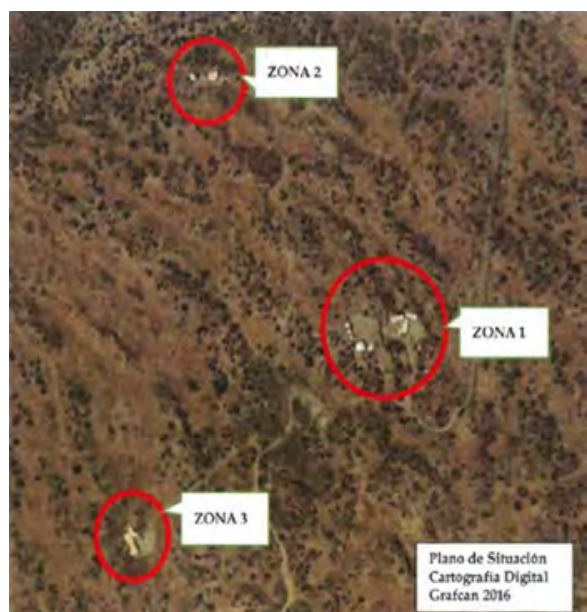

Fig. 17. Vista actual. La Casa del Médico y las Gañanías estuvieron próximas y al oeste de la zona 1.

de La Orotava, para inscribirlas como bienes de propios ${ }^{41}$. Fue este el punto final del largo proceso de desarticulación del régimen comunal en Las Cañadas del Teide ${ }^{42}$.

En el archivo municipal del Ayuntamiento de La Orotava no existe registro alguno de ninguna de las casas próximas a la zona conocida como del Sanatorio -si exceptuamos la única solicitud registrada por Bernardo Benítez de Lugo el 18 de agosto de 1919-. Tampoco hay inscripciones en el Registro de la Propiedad de La Orotava, aunque sí se recogen de modo sucinto y superficial en el plano catastral del Ministerio de Economía y Hacienda.

Se prohíben y eliminan entonces por ley los asentamientos de enfermos llevados a cabo desde la década de 1910 en Las Cañadas del Teide y los alrededores del Sanatorio del Teide -coincidiendo con testimonios orales de Telesforo Bravo Expósito, Wolfredo Wilpret de la Torre e Isidoro Sánchez García-. Pero no sucede así con las construcciones de El Portillo, pues estas quedaron fuera del Parque Nacional.

Tampoco se extrema la vigilancia sobre los alrededores del Sanatorio y las Gañanías, donde aparecerán sorprendente y clandestinamente diferentes construcciones -siendo en la actualidad 13 las casas que permanecen en pie y uso-. Si se observan y comparan las figuras 16 y 17 -fotos del catastro hechas en 1956 por un satélite norteamericano, depositadas en el Archivo Provincial de Tenerife, y las de Grafacan de 2016-, puede afirmarse con absoluta certeza que estas casas son poste-

41 Archivo Municipal de La Orotava. Actas municipales, 29-3-1954 y 11-6-1954.

42 García Mesa, Domingo A., Núñez Pestano, Juan Ramón y Quirantes GonzáLEZ, Francisco: opus cit. p. 142. 
riores al año 1956 -véase igualmente en la fototeca digital del Centro Nacional de Información Geográfica (CNIG), http://pnoa.ign.es/pnoaimagen- y no anteriores.

Otros testimonios orales -además de los citados, conocemos el del aparejador municipal Diego Juan Álvarez, el del notario de la villa Marcos Guimerá Ravina y otros anónimos- coinciden al afirmar que la "Casa del Médico» y el edificio de «Las Gañanías» quedó para el uso y cuidado del médico municipal Buenaventura Machado Melián (1922-2011), quien la usaba personalmente o la cedía a los vecinos de La Orotava que subían durante los veranos para «hacer curas»-sobre todo de afecciones epidérmicas-.

$\mathrm{Al}$ ser estas dos construcciones propiedad del Ayuntamiento de La Orotava -uno de los integrantes principales del Patronato del Parque Nacional del Teide-, fueron demolidas a finales de la década de los años ochenta del pasado siglo. Un problema bien diferente se plantea con las edificaciones actuales, pues si el terreno es del Ayuntamiento, será necesario expropiar y demoler las construcciones. El título de Patrimonio de la Humanidad otorgado por la Unesco a Las Cañadas del Teide en el año 2007 es el principal argumento, pero no el único, para proceder a su desaparición.

Puede afirmarse, por tanto, que estas nuevas casas se construyeron muy rápidamente, entre los años 1956 y los inmediatamente siguientes, antes de que la aplicación de la Ley del Parque Nacional del Teide de 1954 se llevara a cabo con el máximo rigor. Llama la atención, sin duda, la indudable tolerancia tanto de las autoridades del Parque como las del Ayuntamiento de La Orotava por cuanto se refiere a la existencia de dichas construcciones. Los usufructuarios de las mismas parecen ser ciertas personalidades locales de aquella época, por lo que cabe suponer cierta permisividad oficial para con ellos.

\section{SOBRE EL INTERÉS TERAPÉUTICO DE LAS CAÑADAS DEL TEIDE. CONSIDERACIONES ACTUALES}

En el año 2004 se publica el libro El Teide. De Mito Geográfico a Parque Nacional, de Nicolás González Lemus e Isidoro Sánchez García. Por lo que se refiere al asunto que nos ocupa -los asentamientos en Las Cañadas del Teide por causa de la tuberculosis- ${ }^{43}$, los datos que se exponen no ofrecen información relevante de los asentamientos establecidos desde el año 1910 ni después de 1954, ni tampoco explican sus causas. Sin llegar a señalar los criterios de los médicos de la época y los problemas derivados de unos asentamientos fuera del control sanitario, sí concluyen que "por razones presupuestarias, falta de apoyo de las instituciones, inclemencias climáticas, los avances de la farmacopea y el mismo ataque que sufre la cura de

43 González Lemus, Nicolás y Sánchez García, Isidoro (2004): El Teide, de Mito Geográfico a Parque Nacional. Nivaria Ediciones. 


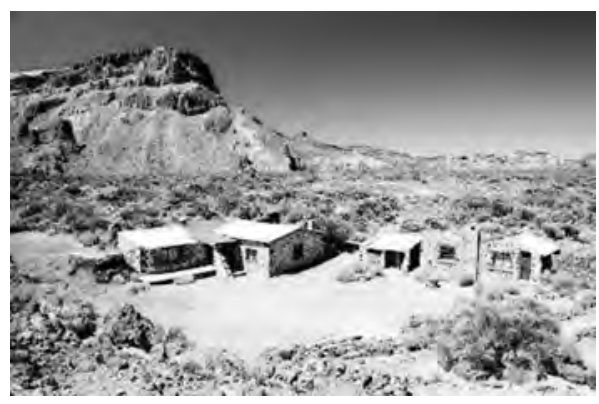

Fig. 18. Vista panorámica de una de las tres agrupaciones de casas.

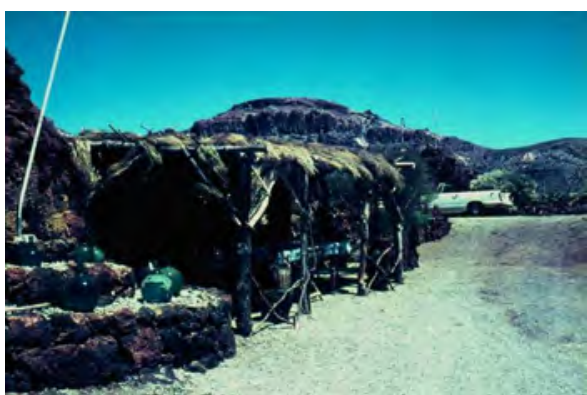

Fig. 19. Una construcción formada y techada con material vegetal. Al fondo La Cañada de La Grieta. Circa 1970.

montaña de elevada altitud por sus fuertes variaciones térmicas cotidianas, nunca se llegó a construir el sanatorio» ${ }^{44}$.

El 8 de noviembre de 2006, en la II Semana Científica Telesforo Bravo, celebrada en el Instituto de Estudios Hispánicos de Canarias, el biólogo y profesor de la Universidad de La Laguna Octavio Rodríguez Delgado dictó una conferencia con el título El paisaje vegetal en Las Cañadas: su transformación por la intervención humana, publicada posteriormente en octubre de 2007. Según explicaba el presidente del IEHC, Nicolás Rodríguez Münzenmaier en la presentación de esta II Semana Científica, «Octavio Rodríguez nos documentó sobre las diferentes actividades que ha realizado el hombre en el Parque Nacional del Teide y los cambios que ha propiciado sobre la vegetación y en el paisaje de Las Cañadas».

Según el mencionado profesor,

en la zona de El Sanatorio lo primero que sorprende en las fotografías es la desaparición de dos grandes edificios: la casa del médico, destruida en un incendio, y las caballerizas, demolidas por acuerdo del Patronato de Parque Nacional. Además, en la primera fotografía se puede apreciar como en los alrededores de la casa del médico no quedaba ninguna retama, salvo una completamente seca en medio del malpaís, mientras que en la actual, la recolonización del retamar es sorprendente, lo que también se aprecia, aunque de forma no tan espectacular, en la segunda fotografía.

Curiosamente, en un artículo que dice ser exhaustivo, pues pasa revista a todo el espacio y el tiempo de Las Cańadas del Teide, no hay mención alguna a las casas circundantes al Sanatorio mencionado.

Otro artículo, firmado por Nicolás González Lemus, Juan Carlos Carracedo Gómez y Manuel Durbán Villalonga, revela muy poca información en rela- 

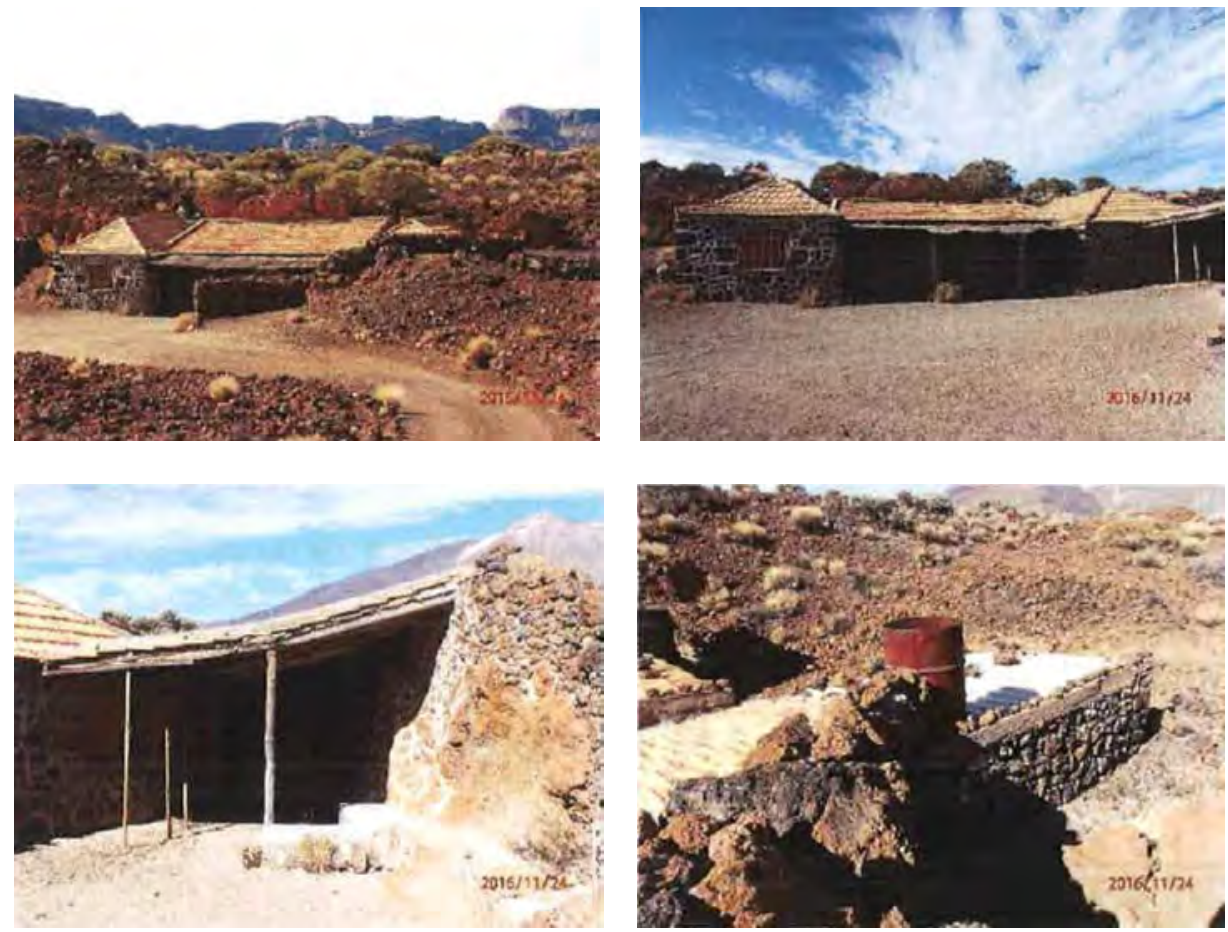

Figs. 20, 21, 22 y 23. Detalles de varias construcciones en la actualidad.

ción con el asunto que nos interesa y ofrece, además, una explicación tan reiterada como incierta. Dice, así, González Lemus que «a lo largo del siglo xx el Teide va a ser reclamo, primero como estación terapéutica por su atmósfera clara, radiación solar intensa y carga iónica del aire» ${ }^{45}$.

Si nos atenemos, por otra parte, a la tesis doctoral de Francisco Javier Castro Molina Arquitectura y medicina en Canarias. Dispositivos asistenciales y recursos sanitarios en Tenerife (ss. XVI-XX) ${ }^{46}$, al margen de algunos datos y comentarios semejantes a los ya recogidos a lo largo de este trabajo, no es posible encontrar ninguna referencia alusiva o relacionada con la utilidad de estas construcciones, tanto las primeras como las actuales, ni como recintos para la curación de enfermedades ni tampoco como patrimonio etnográfico.

45 González Lemus, Nicolás, Carracedo Gómez, Juan Carlos y Durbán Villalonga, Manuel (2009): El Parque Nacional del Teide: Patrimonio mundial de la UNESCO. Anuario de Estudios Atlántico, p. 535.

46 Tesis presentada en el curso 2011-2012, serie Humanidades y Ciencias Sociales de la ULL. 
El trabajo del doctor Emilio González Reimers ${ }^{47}$ Consideraciones teóricas acerca del efecto del clima de Las Cañadas (Tenerife) sobre la tuberculosis y otras afecciones respiratorias aborda y revisa, a la luz de los conocimientos actuales, algunos aspectos muy relevantes sobre la posible influencia del clima de Las Cañadas en el curso evolutivo de la tuberculosis y otras afecciones respiratorias. Entre las conclusiones finales a las que llega el mencionado doctor, establece que «por lo tanto, las condiciones climáticas de este entorno de Las Cañadas del Teide -baja humedad, baja temperatura, hipoxemia- son lo suficientemente adversas para que el ya enfermo de asma, tuberculosis u otro proceso respiratorio crónico experimentara realmente una mejoría, pese a la moda que imperó durante décadas. Otra cosa muy diferente es que en esos sanatorios alpinos y también en Las Cañadas la potente radiación ultravioleta, el calor y la sequedad y sobre todo el no hacinamiento -y probablemente una mejor nutrición, aspecto al que los primeros sanatorios daban una importancia capital-, disminuyeran la contagiosidad y mejoraran el estado general del paciente» ${ }^{48}$.

Un artículo más reciente firmado por Domingo A. García Mesa, Juan Ramón Núñez Pestano y Francisco Quirantes González, profesores de la Universidad de La Laguna, y titulado La lucha por la apropiación de los recursos y el fin de los aprovechamientos comunales en Las Cañadas del Teide: formas de propiedad o sistemas de gestión, suscita varias cuestiones planteadas a lo largo de este estudio. Según señalan estos autores, dígase aquí a modo de resumen:

Durante la posguerra (...) los aprovechamientos tradicionales (pastoreo y carboneo) fueron prohibidos por entrar en conflicto con las medidas conservacionistas impuestas por la administración forestal y resultar contrarias a la protección del paisaje que requería la declaración del Parque Nacional de Las Cañadas del Teide (1954), pero no sucedió lo mismo con otros aprovechamientos que resultaban mucho más rentables como la extracción del cisco de retama. El auge de la exportación de plátanos provocó una creciente demanda de estos productos para las fincas de la costa, por lo que se suprimió el derecho vecinal de aprovechamiento y se implantó el sistema de subastas de efectos forestales para acceder a este recurso, al igual que sucedió con la pinocha en los pinares de la Isla. De igual modo, los intereses económicos de los concesionarios lograron que, a pesar de la declaración del Parque Nacional, continuara durante años la explotación de piedra pómez y rocas y se desarrollasen los proyectos turísticos en Las Cañadas, destacando la instalación del Parador Nacional ${ }^{49}$.

47 Artículo presentado en el Anuario del Instituto de Estudios Canarios n. ${ }^{\circ}$ LIV junto a Arnay de La Rosa, Matilde profesora de la ULL. 2010.

48 Op. cit., p. 233.

49 García Mesa, Domingo A., Núñez Pestano, Juan Ramón y Quirantes González, Francisco: Revista de Historia Canaria n. ${ }^{\circ}$ 197. Opus cit., pp. 97-142. 


\section{CONCLUSIONES}

A partir de las fuentes documentales constatadas pueden distinguirse en el espacio de Las Cañadas del Teide, y hasta el año 1954, dos tipos de asentamientos bien diferenciados.

El primer tipo de asentamientos se refiere a construcciones efímeras y populares - chozas y corrales-, cuya única y exclusiva finalidad -al margen del secular uso dado por los cabreros- fue el alojamiento esporádico y temporal de enfermos que acudían a Las Cañadas para seguir tratamientos relacionados con diversas afecciones -tisiológicas, respiratorias, digestivas y epidérmicas-. Dichos enfermos, a pesar de las prevenciones y advertencias manifestadas por destacados médicos de la época, utilizaron este espacio por varios motivos: por la creencia en las condiciones sobrenaturales de la atmósfera y los manantiales de agua de las cumbres -inciertas pero muy extendidas entre las clases más humildes y ciertas personalidades interesadas-; por la ausencia de recursos farmacológicos y la baratura de medios que suponía la estancia; por la desesperación ante una enfermedad pandémica -en el caso de la tuberculosis, no así en el resto- que no ofrecía curación.

La propaganda de tales condiciones sobrenaturales - tan exageradas como en absoluto contrastadas- fue combatida y desmentida por numerosos y reconocidos médicos de la época. Pero este asunto se entremezcló interesadamente con otros de mayor relieve, como fueron la construcción de la carretera La Orotava-Vilaflor, el «Funicular del Teide», la explotación turística por medio de "Hoteles-Albergues», etc. La creación del Parque Nacional del Teide, como hemos señalado, erradica totalmente tales asentamientos.

El segundo tipo de asentamientos, posterior al año 1954, responde a motivos muy diferentes. Una vez que fue construido el Hospital Antituberculoso de Santa Cruz de Tenerife, germen del actual Hospital Universitario de Canarias, los enfermos de tuberculosis fueron tratados mediante el aislamiento y la administración pautada de antibióticos específicos. Los nuevos asentamientos acabaron sumando trece edificaciones permanentes distribuidas en tres bloques bien diferenciados. Su construcción fue llevada a cabo en el entorno del «Sanatorio», esto es, de la «Casa del Médico» y «Las Gañanías», aprovechando la proximidad del mismo, así como la conducción de aguas que llegaba hasta este. Paradójicamente, su existencia llama la atención por su no apariencia, pues están perfectamente ocultadas por el paisaje. Tales construcciones son posteriores al ańo 1956, como lo demuestran las diferentes fotos aéreas hechas por satélite. Se trata, por consiguiente, de unos asentamientos que no respondían ya a las necesidades médico-terapéuticas como sí sucede en el caso de los primeros.

La creación del Parque Nacional del Teide en el año 1954, y la legislación restrictiva que acompañó al mismo, hace más sorprendente la aparición de estas edificaciones. Sin embargo, como se ha señalado, el real decreto de creación del Parque Nacional no acabó con algunas de las prácticas extractivas del pasado -aprovechamiento de cisco de retama, extracción de piedra pómez y de rocas para construcción, etc.-. Por ello cabe presuponer que cierta permisividad o indeterminación por parte de las autoridades de la época fuera aprovechada por algunas personas de rela- 
tiva posición social y económica de La Orotava. La brevedad del tiempo y la premura para su rápida construcción así parecen confirmarlo.

Finalmente y teniendo en cuenta lo expuesto, puede afirmarse que si los primeros asentamientos, utilizados en la etapa anterior al año 1954, pueden poseer algún valor de carácter etnográfico o como patrimonio histórico, dada su primigenia función terapéutica, en el caso de las actuales construcciones dicho carácter o condición es absolutamente inexistente. Estos son aspectos a tener en gran consideración a la hora de adecuar un territorio declarado Patrimonio de la Humanidad por la Unesco.

Recibido: 21-03-2019. Aceptado: 11-04-2019 
